BMC

Ecology

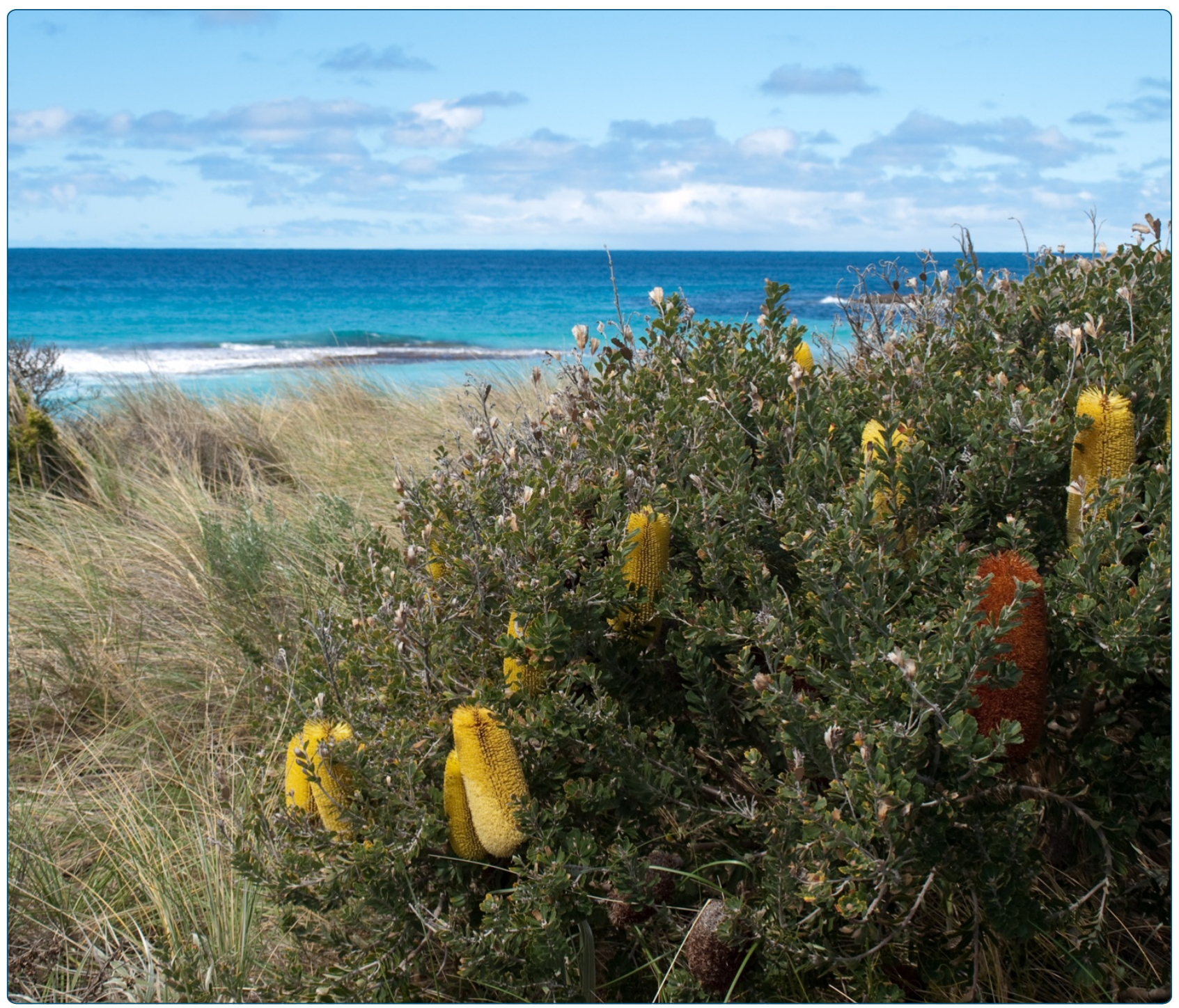

The phylogenetic signal of species co-occurrence in high-diversity shrublands: different patterns for fire-killed and fire-resistant species

Cardillo

() Biomed Central 


\title{
The phylogenetic signal of species co-occurrence in high-diversity shrublands: different patterns for fire-killed and fire-resistant species
}

\author{
Marcel Cardillo
}

\begin{abstract}
Background: Using phylogenies in community ecology is now commonplace, but typically, studies assume and test for a single common phylogenetic signal for all species in a community, at a given scale. A possibility that remains little-explored is that species differing in demographic or ecological attributes, or facing different selective pressures, show different community phylogenetic patterns, even within the same communities. Here I compare community phylogenetic patterns for fire-killed and fire-resistant Banksia species in the fire-prone shrublands of southwest Australia.
\end{abstract}

Results: Using new Bayesian phylogenies of Banksia, together with ecological trait data and abundance data from 24 field sites, I find that fire regeneration mode influences the phylogenetic and phenotypic signal of species co-occurrence patterns. Fire-killed species (reseeders) show patterns of phylogenetic and phenotypic repulsion consistent with competition-driven niche differentiation, but there are no such patterns for fire-resistant species (resprouters). For pairs of species that differ in fire response, co-occurrence is mediated by environmental filtering based on similarity in edaphic preferences.

Conclusions: These results suggest that it may be simplistic to characterize an entire community by a single structuring process, such as competition or environmental filtering. For this reason, community analyses based on pairwise species co-occurrence patterns may be more informative than those based on whole-community structure metrics.

Keywords: Coexistence, Competition, Co-occurrence matrix, Phylogenetic community ecology, Phylogenetic conservatism, Regeneration strategy, Southwestern Australia

\section{Background}

Phylogenetic information is now commonly employed in analyses of species co-occurrence and community assembly, as a way of explaining patterns of species distributions and diversity [1-4]. Community phylogenetic studies have increased steadily in sophistication over the past decade. For example, the effects of spatial and phylogenetic scale have been explored [5-7], and null models for phylogenetic community structure have been developed [6,8]. More generally, the idea that patterns of phylogenetic attraction (where close relatives are more likely to co-occur than expected by chance) and repulsion (where close relatives

Correspondence: marcel.cardillo@anu.edu.au

Centre for Macroevolution and Macroecology, Research School of Biology, Australian National University, Canberra A.C.T. 0200, Australia are less likely to co-occur) can act as a proxy for ecological processes is now regarded more critically. It is generally agreed that phylogenetic and phenotypic patterns should be analysed simultaneously if informative inferences about processes are to made [6].

One assumption that remains common in community phylogenetics, however, is the idea that the structure of a community is governed by an overarching process, such as competition or environmental filtering, that applies to all species in the community. This assumption is implicit in whole-community metrics of phylogenetic community structure such as NRI and NTI [4]. While some studies provide evidence for the simultaneous operation of competition and environmental filtering, often depending on spatial or phylogenetic scale [5-7], the assumption that the same process applies to all species in a community, at a 
given scale, is typically still made. However, ecological processes such as competitive exclusion occur at the level of individuals, populations or species, not communities. The structure of a community is simply an emergent property that results from the influence of such processes on the combined distributions of multiple species within a region. Therefore, it is possible that whole-community metrics obscure much informative variation in phylogenetic structure by "averaging out" the patterns of occurrence or abundance of different species within a community. An alternative approach is to analyse the phylogenetic and phenotypic signal of pairwise co-occurrences among species across a set of communities (e.g. [9-11]. This approach treats species pairs, rather than communities, as the units of analysis, more easily allowing patterns of cooccurrence to be examined separately for species with different demographic and ecological attributes.

In this study I compare the phylogenetic and phenotypic signal of co-occurrence patterns of plant species that differ in fire-regeneration strategy, in the Mediterranean-climate shrublands of southwestern Australia. This ecosystem type is exceptionally species-rich, but is still poorly represented among community phylogenetic studies (but see [1113]. In these shrublands, fires are frequent, with average recurrence intervals around 10-15 years [14]. Some authors have argued that in these highly disturbed, nonequilibrium communities, classic theories of species coexistence through niche differentiation are less realistic than models of coexistence based on lottery recruitment and the availability of transient niches [14-19]. Other studies in Mediterranean shrublands have found evidence for patterns that may be competition-driven, such as niche differentiation [20] or phylogenetic overdispersion [11].

There are two main strategies for coping with fire within Mediterranean-climate floras. Resprouters survive fire and regenerate from lignotubers, epicormic buds or other structures, and reseeders are killed by fire and replaced by seedlings. Although there are different degrees and modes of resprouting, in Mediterranean-climate shrublands regeneration mode is usually considered a simple dichotomous variable [21]. Essentially, therefore, the flora is divided into two components with fundamentally different demographics: fire-resistant (resprouter) populations are relatively stable, persistent and impervious to disturbance by fire, and fire-killed (reseeder) populations are more variable and susceptible to local extinction after fire [18,21,22].

Do these ecological differences between resprouters and reseeders generate different patterns of association between phylogenetic relatedness, phenotypic similarity, and co-occurrence? Several predictions can be made:

(1) If resprouters are longer-lived with more stable populations, co-occurrence among resprouter species may be influenced by competition for space, soil moisture or nutrients, and by differentiation of species along niche axes associated with the preemption of these resources. Among reseeders, on the other hand, co-occurrence may be mediated by the frequency of fire, and its influence on dispersal and colonization, rather than by niche differentiation [22,23]. Under this scenario we would predict phylogenetic and phenotypic repulsion among resprouters, but not among reseeders.

(2) Alternatively, competition may be intense among reseeders, because the pressure to grow rapidly to maturity and set seed within the average fire interval is traded off against the costs of faster growth [24,25]. Hence, co-occurrence among reseeders may be controlled by niche differentiation along life-history axes associated with time to maturity, or environmental gradients such as soil fertility, which influences growth rates [22]. Under this scenario, we would predict phylogenetic and phenotypic repulsion among reseeder species but not among resprouters.

(3) Finally, there may be no difference in the mechanisms of co-occurrence between species differing in regeneration mode, if co-occurrence is determined primarily by processes that apply regardless of regeneration mode.

As a case study for testing these predictions I use the genus Banksia, one of Australia's iconic plant genera and a prominent part of the flora of southwestern Australia. The genus includes 170 species that have radiated into a variety of growth forms, from prostrate ground-covers to shrubs and trees $>6 \mathrm{~m}$ in height, and includes resprouters and reseeders. Local-scale diversity of Banksia is high: up to twelve species have been recorded from single $10 \mathrm{~m} \mathrm{x}$ $10 \mathrm{~m}$ plots [26]. The analyses presented here are based on surveys of species within plots of two sizes: $20 \mathrm{~m} \mathrm{x} 20 \mathrm{~m}$ (0.04 ha) and $200 \mathrm{~m} \times 200 \mathrm{~m}$ (4 ha). I begin by quantifying patterns of co-occurrence among pairs of Banksia species within these plots. I then test whether cooccurrence among species pairs is associated with phylogenetic relatedness, regeneration mode, ecological similarity, and similarity in soil type preferences. I interpret co-occurrence patterns in the context of the phylogenetic signal in the different niche dimensions.

\section{Methods}

\section{Banksia surveys}

Banksia species were surveyed at 24 shrubland sites spread across an arc of the Southwest Botanical Province (SWBP) spanning approximately $250 \mathrm{~km}$ (Figure 1). At each site, a $200 \mathrm{~m}$ x $200 \mathrm{~m}$ (4 ha) plot was established and surveyed for the presence of Banksia species. Each 


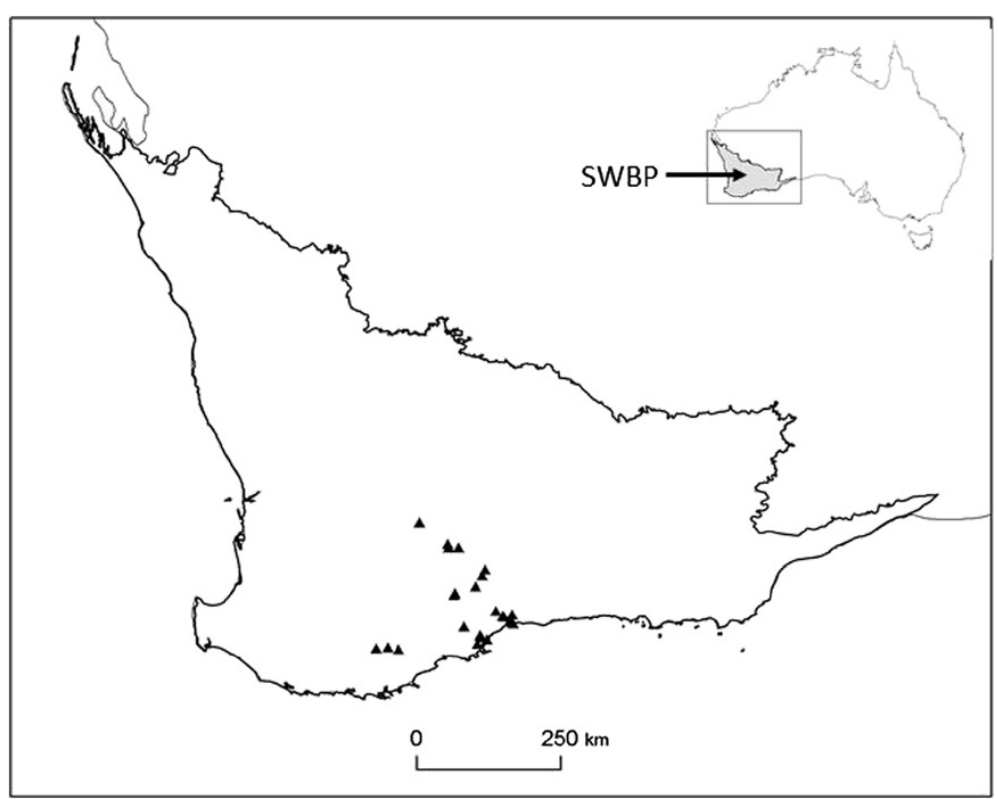

Figure 1 Map of the SWBP showing the 24 study sites (triangles).

4 ha plot was divided into four quadrants, and one $20 \mathrm{~m} \mathrm{x}$ $20 \mathrm{~m}(0.04 \mathrm{ha})$ plot was randomly placed within each quadrant. In each 0.04 ha plot, the presence and abundance (number of individual plants) of each Banksia species was recorded. The survey data thus comprised species presences within 24 large plots and abundances within 96 small plots. Species abundances were estimated for each 4 ha plot by taking the mean value across the four 0.04 ha plots within it. The species $\mathrm{x}$ sites matrices are provided in Additional file 1.

\section{Phylogeny reconstruction}

The phylogeny used in this study is a subset pruned from a larger phylogeny of 198 Banksia taxa. This phylogeny was constructed from a combination of chloroplast DNA sequences previously available on GenBank (88 taxa) and newly-generated sequences (110 taxa). Most of the newly-generated sequences are for species of the former genus Dryandra which has recently been merged with Banksia [27], but has not previously been the subject of a complete phylogenetic study. The complete phylogeny of 198 taxa, with a full description of the methods of DNA extraction and sequencing, and phylogeny reconstruction, will be published elsewhere (Cardillo \& Pratt, unpublished ms), but below I present a summary of the methods.

Total genomic DNA was extracted from tissue samples of both fresh material and dried herbarium specimens for 110 Banksia taxa (including full species, subspecies and varieties), using a Qiagen DNEasy Plant Mini Kit according to the manufacturer's protocol. Sequencing was carried out at Macrogen, Seoul, Korea; 3656 bp of sequences across five non-coding chloroplast regions (rpl16 intron , $\mathrm{psbA} / \operatorname{trnH}$ spacer, trnL intron, trnL/trnF spacer, trnT/trnL spacer) were obtained. The 110 new sequences were combined with the 88 sequences from Genbank, then aligned manually using Geneious v5.6.3 [28]. The phylogeny was estimated together with divergence times using a Bayesian analysis in BEAST 1.6.2 [29]. Calibrations were based on ages of the Banksia crown (42Mya, lognormal prior) and stem (62Mya, lognormal prior) estimated from fossil data [30]. To constrain the age of the root node, a normal prior with mean 77Mya was used, based on the estimated divergence time between the lineages leading to Banksia and Hakea / Grevillea in a recent genus-level phylogeny of the Proteaceae [31]. A Yule prior was used for the speciation process, and an uncorrelated lognormal model for variation in rate of molecular evolution [32]. The function modelTest() in the $\mathrm{R}$ library phangorn [33] was used to choose an $\mathrm{HKY}$ + gamma substitution model. Two separate MCMC chains were run for $>40,000,000$ generations, sampling trees every 10,000 generations. Sampling adequacy was diagnosed by examining effective sample sizes (ESS) using Tracer 1.5 [29]; the runs were terminated when all parameter values had ESS $>300$.

The resulting 198-taxon phylogenies include 34 of the 39 Banksia taxa found in the field plots; the maximum clade credibility tree, pruned to include only these 34 taxa, is shown in Figure 2. To account for phylogenetic uncertainty, most of the phylogeny-based analyses I present here were repeated on 1000 trees sampled evenly from the Bayesian posterior distribution, and I present results as summary values of distributions from this sample. 


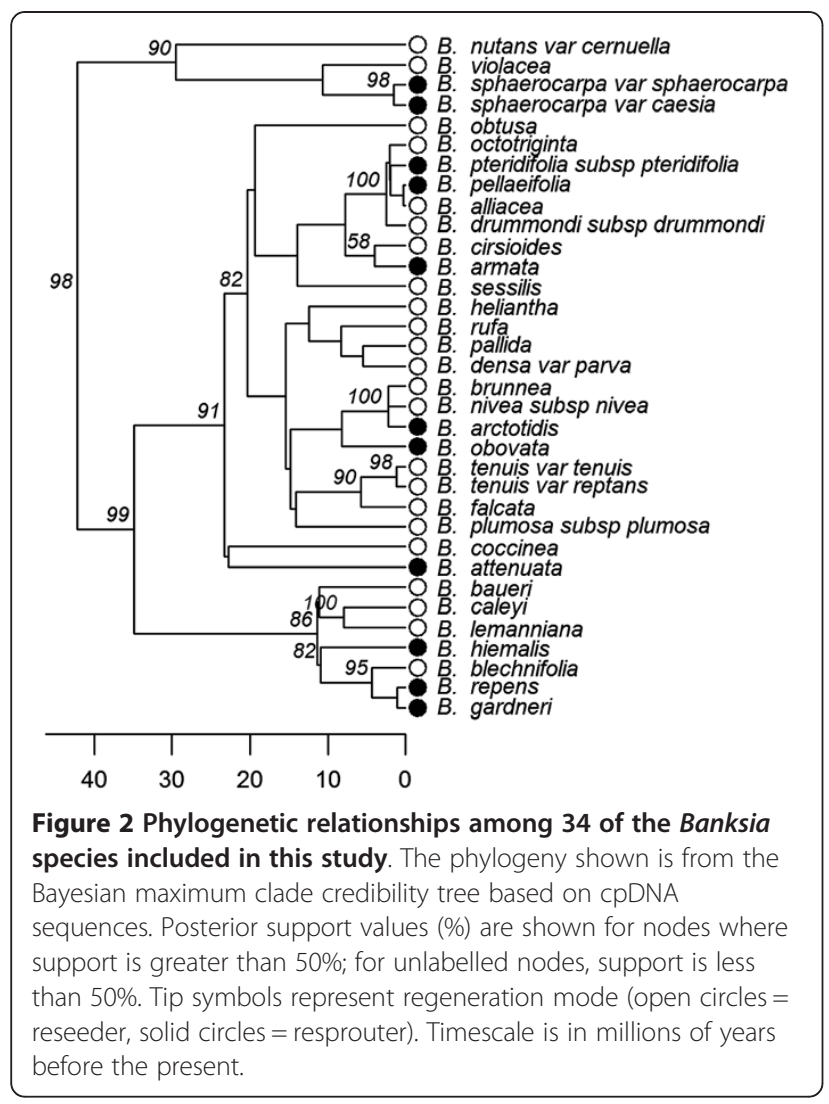

\section{Ecological and environmental data}

I compiled a dataset of ecological traits for all Banksia species (see Additional file 2) using information from the standardized species entries in the Flora of Australia $[34,35]$ and in The Dryandras [36]. Data for the following variables were recorded: (1) maximum height (species listed as prostrate were given a height of $0.2 \mathrm{~m}$ ); (2) mean seed length; (3) fire regeneration mode (resprouter /reseeder); (4) months of the year in which flowering occurs. Height and seed size are both key indicators of major axes of variation in life history strategies of plant species [37-39], and the timing of flowering may be involved in mediating competition and structuring communities $[40,41]$.

Many SWBP species display a high degree of edaphic specialization [42], and the fine-scale mosaic of soil types is suspected to play a role in generating and maintaining the region's high species richness $[14,43]$. I therefore focused on edaphic gradients to analyse the influence of environmental filtering. In each 0.04 ha plot I collected four soil samples, which were aggregated and analysed by the Environmental Analysis Laboratory, Southern Cross University, for concentrations of calcium, potassium, nitrate, phosphorus and silicon, and for $\mathrm{pH}$ and organic matter content (see Additional file 3).
Accounting for non-overlapping geographic distributions Where species have originated allopatrically and remained geographically separated, metrics of co-occurrence of species across a set of sites could detect significant negative co-occurrences that may simply be the result of speciation history rather than species interactions or other community assembly processes. This is likely to be common in a group such as Banksia, in which a large proportion of species have highly restricted geographic distributions. To minimize this effect I omitted from the analyses pairs of species with no overlap in geographic distribution. Geographic distributions of species were inferred from georeferenced herbarium records obtained from the Australian Virtual Herbarium (http://chah.gov.au/avh/). I removed records with clearly erroneous location data (e.g. in the sea) or those well outside a species' distribution limits indicated in FloraBase [44]. I then obtained an estimate of the extent of occurrence of each species by using ArcGIS [45] to draw a minimum convex polygon around the set of record locations. These are not intended to be highly resolved reconstructions of the boundaries of species' distributions; they are simply approximations which I used only to judge whether pairs of species overlap geographically. I omitted species pairs with no overlap from further analyses of distance matrices.

\section{Co-occurrence of species pairs}

After omitting non-overlapping species pairs, the 39 species of Banksia provided 419 pairwise species comparisons. For each pair, I calculated Schoener's index of cooccurrence $\mathrm{C} i j$, a measure of co-occurrence that takes into account the presence or absence of species at each site, as well as their proportional abundances [46]. Cooccurrence was calculated for both plot sizes (0.04 ha and $4 \mathrm{ha}$ ), and separately for species pairs where both species are reseeders (174 pairs), where both are resprouters (48 pairs), and where the two species differ in regeneration mode (197 pairs). The calculation of Cij was done using the "species.dist" function in the $\mathrm{R}$ library Picante [47].

\section{Associations between co-occurrence, phylogenetic and ecological distance}

Of the 39 Banksia species found in the field plots, 34 were included in the phylogeny. Phylogenetic distances among these 34 species were measured as the total length of the branches connecting each pair of species. Differences in height, seed size and length of the flowering period were calculated among all of the 39 species in the field plots. To measure overlap in flowering periods for each species pair, I calculated the number of months in which both species flower, and divided this by the shorter of the lengths (in months) of the flowering periods of the two species. I then subtracted this value from 
one so that a larger value corresponds to greater dissimilarity in flowering period, for consistency with the other measures of ecological distance. To quantify species' edaphic preferences, I calculated linear regressions of species' log-transformed abundances against the values of each soil type variable, across the 96 small plots. Following Helmus et al. (2007) [8] I took the slopes of these regressions, whether or not they differed significantly from zero, as a measure of each species' association with each soil type variable. The distances between species in these variables were simply the differences in the regression slopes.

All tests for associations among pairwise distance values were done using quantile regression with bootstrapped p-values. Because each species contributes to multiple species pairs, degrees of freedom are inflated and $\mathrm{p}$-values based on standard significance tests are misleading. Bootstrapping avoids this problem by randomizing the dataset and testing significance by comparing $\mathrm{p}$-values to the distribution of random p-values. I tested for associations between co-occurrence and phylogenetic distance, and separately for associations between co-occurrence and each ecological and edaphic distance measure. These tests were done for both 0.04 ha and 4 ha plot sizes, and for resprouters, reseeders and resprouter vs. reseeders separately. Quantile regressions were done using the "quantreg" library in R (http://cran.r-project.org/ web/packages/quantreg).

\section{Measuring phylogenetic signal in ecological traits and edaphic preferences}

Associations between co-occurrence and phylogenetic, ecological and edaphic distances can only be properly interpreted if we know the degree to which ecological traits and edaphic preferences are phylogenetically conserved or labile. To measure the strength of phylogenetic signal in height, seed size, length of flowering period and edaphic preferences, I used $\lambda$, a branch-length transformation parameter of the phylogenetic GLS model [48]. I tested the maximum-likelihood estimate of $\lambda$ against hypotheses of $\lambda=0$ (no phylogenetic signal) and $\lambda=1$ (phylogenetic signal consistent with Brownian motion model of trait evolution). To test phylogenetic signal in regeneration strategy, I used the D statistic, which is appropriate for use with binary traits [49]. The $\lambda$ and D statistics were calculated using the "lam.test.single" and "phylo.d" functions in the R library caper (http://rforge.r-project.org/projects/caper). To measure phylogenetic signal in the overlap in species' flowering periods, I used quantile regression to test for significant associations with phylogenetic distance across species pairs.

I then looked for evidence that recently-separated lineages have diverged rapidly with respect to each niche axis. To do this, I plotted the ages of nodes in the Banksia phylogeny against standardized contrasts in the ecological and soil-preference traits at each node. Typically, contrasts are standardized to a common variance by dividing their absolute value by the square root of the variance expected under a Brownian motion model of trait evolution, which corresponds to the sum of the branch lengths at each node [50]. However, particularly for labile traits, this can result in overstandardization, where contrasts become smaller at deeper nodes because they are being divided by a larger number [51]. To minimize this effect, Garland et al. (1992) recommended transforming the branch lengths of the tree prior to standardizing contrasts. Here I transform branch lengths by raising them to a power $\mathrm{k}$, then optimizing the value of $\kappa$ to minimize the correlation between absolute standardized contrasts and their standard deviations [51]. The resulting plots give a visual guide to the patterns of ecological divergence between lineages in each variable. Significant positive outliers on these plots can be interpreted as lineages that have diverged more than expected from their age of separation. Because the branch-length optimization renders slope estimates non-comparable, I applied this test only to the maximum clade credibility tree from the Bayesian analysis, not to the sample of 1000 posterior trees. Contrasts were calculated using the "crunch" function in the R library caper (http://cran.rproject.org/web/packages/caper), and functions written by A. Purvis (unpublished).

\section{Results}

Across all geographically-overlapping species pairs, there is a positive association between phylogenetic distance and the co-occurrence metric Cij (Table 1). This indicates a general pattern of phylogenetic repulsion, with co-occurrence less likely among closely-related species. When resprouters and reseeders are tested separately, phylogenetic repulsion is evident among pairs of reseeder species, but there is no significant association among the resprouter species (Table 1). Among species pairs that differ in regeneration mode, the positive association between phylogenetic distance and $\mathrm{C} i j$ remains significant.

There are positive associations between $\mathrm{C} i j$ and plant height, for all species pairs and for reseeders only, but no association for resprouters only, or for reseeders vs. resprouters (Table 1). This indicates phenotypic repulsion among reseeders, such that pairs of species of similar height are less likely to co-occur. There are no significant associations between co-occurrence and seed size, length of the flowering period or degree of overlap in the flowering period (Table 1).

Among the soil type variables, nitrate and $\mathrm{pH}$ show negative associations with $\mathrm{C} i j$ across all species pairs (Table 2). These associations are not significant among 
Table 1 Slopes of quantile regressions for phylogenetic and ecological distance

\begin{tabular}{llllllll}
\hline Data subset & Plot size (ha) & d.f. & Phylogenetic distance & Height & Seed size & $\begin{array}{c}\text { Flowering period } \\
\text { length }\end{array}$ & $\begin{array}{c}\text { Flowering period } \\
\text { overlap }\end{array}$ \\
\hline all species pairs & 0.04 & 417 & $0.003(0.002,0.004)^{* * *}$ & 0.03 & 0.001 & 0.01 & -0.02 \\
resprouters only & 4 & 417 & $0.004(0.002,0.006)^{* *}$ & $0.06^{* *}$ & 0.002 & 0.02 & -0.07 \\
& 0.04 & 46 & $-0.003(-0.006,0.003)$ & -0.01 & -0.003 & -0.03 & 0.03 \\
reseeders only & 4 & 46 & $-0.006(-0.01,0.003)$ & -0.01 & -0.003 & -0.03 & 0.05 \\
& 0.04 & 172 & $0.003(0.002,0.005)^{*}$ & $0.08^{* * *}$ & 0.0003 & 0.03 & -0.03 \\
resprouters vs. reseeders & 4 & 172 & $0.004(0.003,0.007) \dagger$ & $0.08^{* * *}$ & 0.0008 & 0.03 & -0.04 \\
& 4 & 195 & $0.002(0.001,0.003)^{*}$ & -0.005 & 0.003 & 0.009 & -0.05 \\
\end{tabular}

Response variable is co-occurrence among species pairs. Slope values for phylogenetic distance are medians (with $2.5^{\text {th }}$ and $97.5^{\text {th }}$ percentiles) from 1000 trees sampled from the Bayesian posterior distribution.

${ }^{* * *} p \leq 0.001 ;{ }^{* *} p \leq 0.01 ;{ }^{*} p \leq 0.05 ;+p \leq 0.1$.

resprouters only or reseeders only, but are significant among species pairs that differ in regeneration mode. This indicates a pattern of environmental attraction among species that differ in regeneration mode: species are more likely to co-occur if they are similar in their response to soil nitrate concentration or $\mathrm{pH}$.

Tests for phylogenetic signal in ecological traits (Table 3) show that plant height and seed size are phylogenetically conserved, with median maximum likelihood estimates of $\lambda$ not significantly different from a Brownian model $(\lambda=1)$, while the length of the flowering period is labile (median $\lambda \approx 0$ ). Regeneration mode is labile, with median values of $\mathrm{D}$ rejecting a Brownian model $(D=0)$. The timing of the flowering period, as measured by quantile regression of flowering period overlap $\mathrm{x}$ phylogenetic distance across species pairs, is labile (slope $\approx 0$ and $p \approx 1$ for all 1000 trees). Species preferences in all edaphic variables, with the exception of organic matter, are phylogenetically labile (Table 3 ), with median maximum likelihood estimates of $\lambda$ not significantly different from $\lambda=0$, and significantly different from $\lambda=1$. Preference for organic matter content, on the other hand, appears to be conserved (Table 3).
Plots of node ages against standardized contrasts provide little evidence that closely-related lineages have diverged rapidly with respect to ecological traits or edaphic preferences (Figures 3 and 4). Although many of the slopes of these plots are negative, there are few significant outliers, and few nodes for which divergences are greater than expected from their ages.

\section{Discussion}

The degree to which the high species richness of Mediterranean-climate shrublands is maintained by competition-driven differentiation of functional traits $[11,20]$, or by lottery recruitment and disturbancemediated coexistence $[14,52]$, is not fully clear. These two models make different predictions about the associations between species co-occurrence, phylogenetic relatedness, and phenotypic similarity. I have shown that for Banksia in southwestern Australia, these associations vary between fire-killed and fire-resistant species, suggesting that the ecological processes that govern cooccurrence and community structure do not necessarily apply uniformly to all species within the same communities.

Table 2 Slopes of quantile regressions for soil type preferences

\begin{tabular}{lccccccccc}
\hline Data subset & Plot size (ha) & d.f. & Calcium & Potassium & Phosphorus & Nitrate & pH & Organic matter & Silicon \\
\hline all species pairs & 0.04 & 417 & 10.86 & -30.93 & -0.53 & $-13.25^{*}$ & $-1.49^{* * *}$ & -0.93 & -24.37 \\
& 4 & 417 & 108.1 & -68.25 & -1.08 & $-16.56^{*}$ & $-2.25^{* * *}$ & 0.66 & -81.15 \\
resprouters only & 0.04 & 46 & 134.8 & $-270.41^{*}$ & -0.56 & -18.45 & -1.75 & -4.35 & -284.31 \\
& 4 & 46 & 228.11 & -270.41 & -0.97 & -18.45 & -1.75 & -4.46 & -361.93 \\
reseeders only & 0.04 & 172 & 110.19 & -18.64 & -0.7 & 2.72 & -1.84 & 0.83 & 17.91 \\
& 4 & 172 & 62.91 & -43.89 & -1.3 & -5.47 & -2.49 & 1.04 & -77.57 \\
resprouters vs. reseeders & 0.04 & 195 & -83.04 & -47.1 & -0.49 & $-17.63^{*}$ & $-1.41^{*}$ & -0.95 & -36.73 \\
& 4 & 195 & 35.39 & -94.18 & -0.79 & $-30.97^{* *}$ & $-2.5^{*}$ & & 0.56 \\
\hline
\end{tabular}

Response variable is co-occurrence among species pairs. ${ }^{* *} p \leq 0.001 ;{ }^{* *} p \leq 0.01 ;{ }^{*} p \leq 0.05 ;+p \leq 0.1$. 
Table 3 Phylogenetic signal in ecological traits and edaphic preferences

\begin{tabular}{llll}
\hline & Test statistic & $\mathbf{p}($ labile $)$ & $\mathbf{p}($ Brownian) \\
\hline Ecological traits & & & \\
height & $\lambda=0.98(0.94,1)$ & $0(0,0)$ & $0.25(0,1)$ \\
seed size & $\lambda=0.96(0.84,1)$ & $0(0,0)$ & $0.07(0,1)$ \\
regeneration mode & $D=0.9(0.57,1.16)$ & $0.35(0.09$, & $0.02(0.001,0.11)$ \\
& & $0.68)$ & \\
flowering period & $\lambda=0.0001(0.0001,1)$ & $1,(0.009,1)$ & $0.004(0,1)$ \\
length & & & \\
Soil preferences & & & \\
calcium & $\lambda=0.0001(0.0001,0.98)$ & $1,(0.15,1)$ & $0.008(0,1)$ \\
potassium & $\lambda=0(0,0)$ & $1,(1,1)$ & $0(0,0.01)$ \\
phosphorus & $\lambda=0.0001(0.0001,09)$ & $1,(0.06,1)$ & $0.0001(0,0.16)$ \\
nitrate & $\lambda=0(0,0)$ & $1,(1,1)$ & $0(0,0.004)$ \\
pH & $\lambda=0(0,0)$ & $1,(1,1)$ & $0(0,0)$ \\
organic matter & $\lambda=0.99(0.0001,1)$ & $0.001,(0,1)$ & $1(0.002,1)$ \\
silicon & $\lambda=0(0,0)$ & $1,(1,1)$ & $0(0,0.03)$ \\
\hline
\end{tabular}

For regeneration mode the test statistic is $D$, for all other variables the test statistic is $\lambda$. Values shown are medians (with $2.5^{\text {th }}$ and $97.5^{\text {th }}$ percentiles) from 1000 trees sampled from the Bayesian posterior distribution.
The three scenarios entertained in the Introduction were that (1) patterns for resprouters but not reseeders are consistent with niche differentiation; (2) patterns for reseeders but not resprouters are consistent with niche differentiation; (3) there are no differences in patterns between resprouters and reseeders. The results provide greatest support for scenario 2, with reseeders, but not resprouters, showing patterns of phylogenetic and phenotypic repulsion consistent with niche differentiation. Of the traits included in this study, maximum adult height appears to play a role in driving the phylogenetic signal of co-occurrences among reseeders, as it shows a pattern of phenotypic repulsion and is strongly phylogenetically conserved. In contrast, the results provide no evidence that seed size, or the length or degree of overlap in flowering period, are associated with patterns of co-occurrence. The adult height attained by a plant species represents a tradeoff between the benefits of greater height, such as greater light interception and seed production, and the costs, such as greater proportional investment in non-reproductive support tissue $[24,25]$. Height is therefore a key dimension of ecological variation in plants [38] and a potential axis for niche differentiation where competition between species exists $[53,54]$. The plots of contrasts in height against node
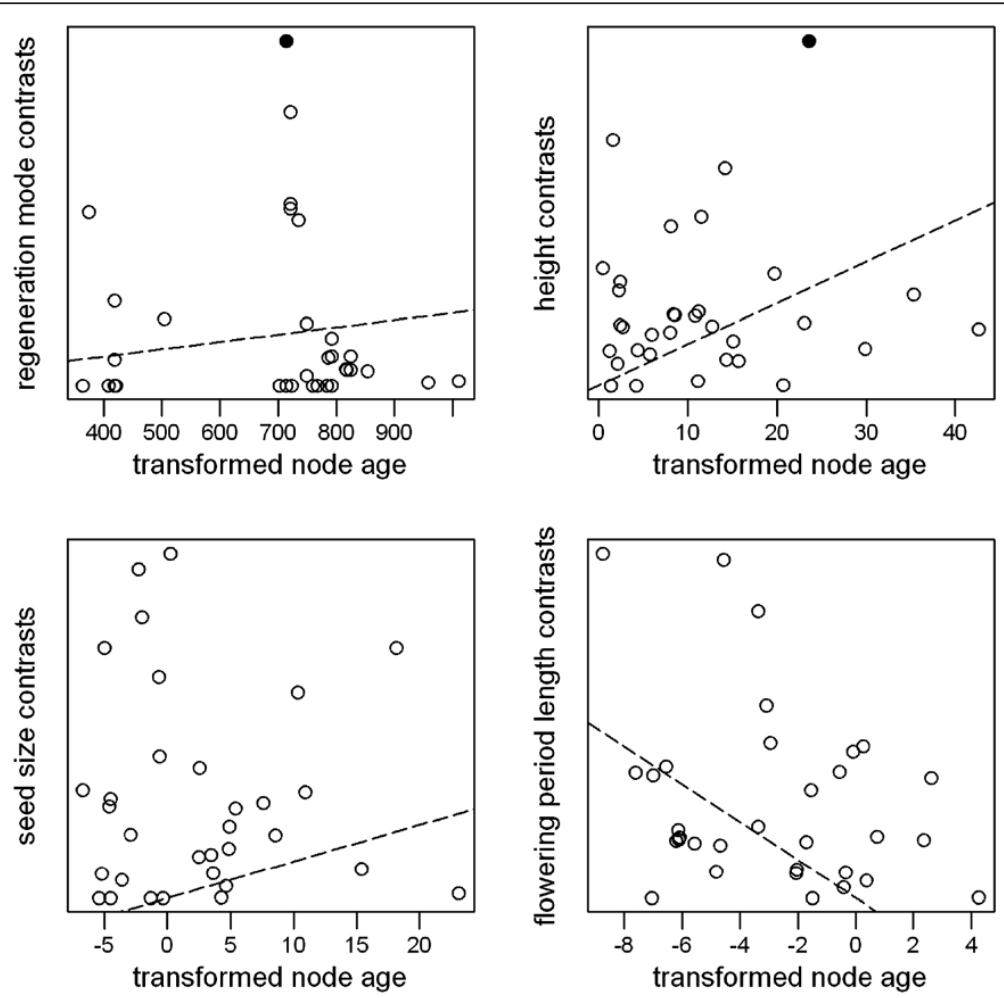

Figure 3 Plots of node ages against standardized contrasts in ecological traits. Node ages are based on transformed branch lengths; see text for details. Points are nodes in the Banksia phylogeny; dashed lines are least-squares regressions through the origin; solid circles are significant outliers from the regression line (absolute studentized residual > 3), indicating nodes with a greater divergence in a given trait than expected from their age. 

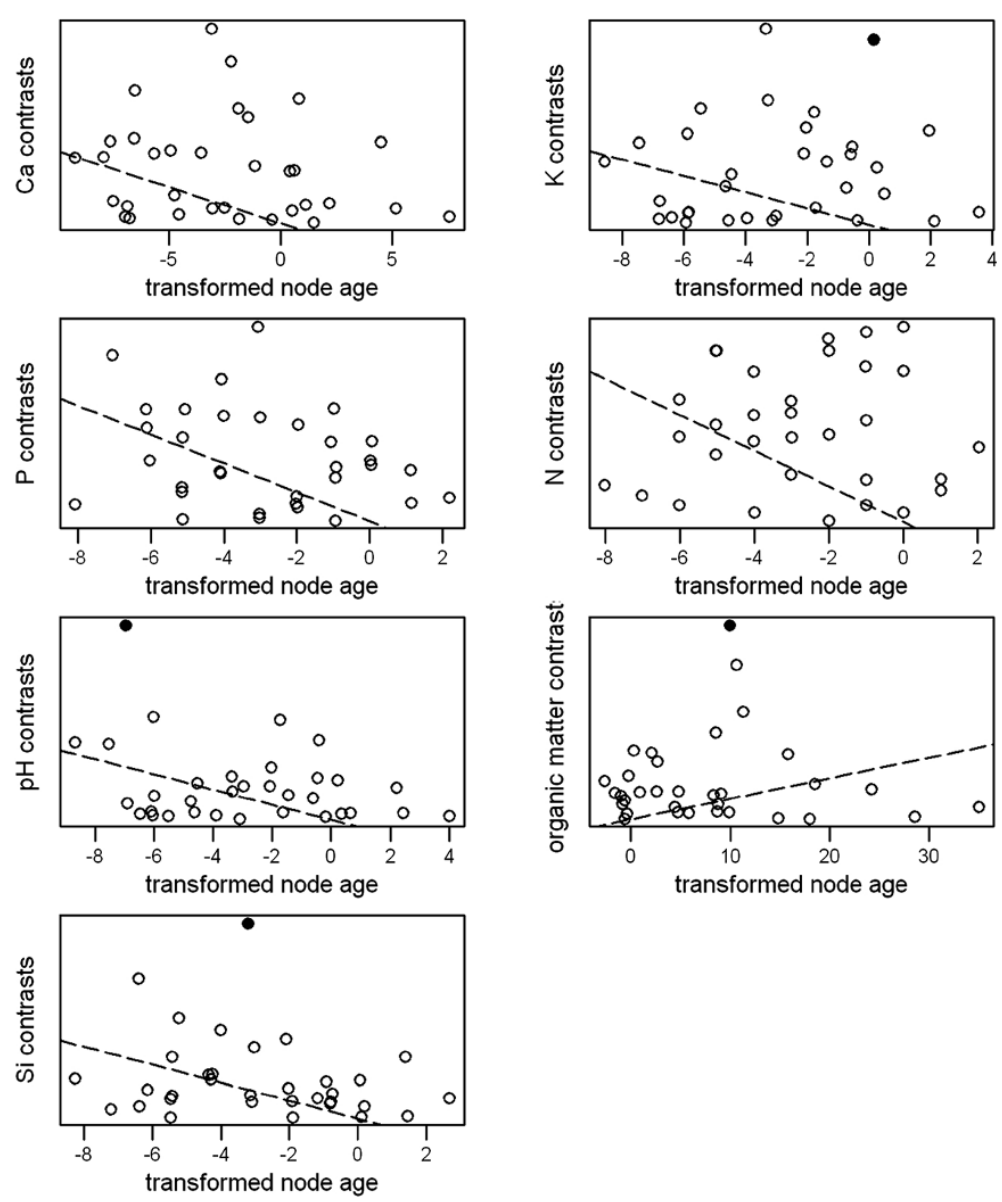

Figure 4 Plots of node ages against standardized contrasts in species' edaphic preferences. Node ages are based on transformed branch lengths; see text for details. Points are nodes in the Banksia phylogeny; dashed lines are least-squares regressions through the origin; solid circles are significant outliers from the regression line (absolute studentized residual $>3$ ), indicating nodes with a greater divergence in a given edaphic preference than expected from their age.

ages show no evidence for rapid divergence among closely-related lineages. Height therefore appears to influence the co-occurrence patterns of reseeding Banksia species by ecological sorting (i.e. competitive exclusion) rather than through microevolutionary divergence.

Why should there be evidence of niche differentiation along the axis of height for reseeders, but not for resprouters? There is likely to be selective pressure on reseeder species to grow rapidly and reach full seed production within the average fire recurrence period, favouring a lower adult height [55]. This is traded off against the costs of faster growth and lower adult height, including greater soil nutrient requirements, lower total seed production and reduced seed dispersal capacity $[22,25,53]$. On the other hand, it is less easy to explain why these selective pressures should be absent in resprouters. It has been observed that some resprouting Banksia species have very low rates of recruitment from seed, and most plants present after a fire are adults that have resprouted, rather than new recruits $[23,55]$. Furthermore, adult height does not reflect longevity in resprouters as it does in reseeders. Hence, the costs and benefits of rapid growth and lower height may be less critical for resprouters compared to reseeders. Of course, it is also possible that because of the smaller number of pairs of resprouter species (48 compared to 174 reseeder pairs), there was simply less power to detect patterns of phylogenetic and phenotypic repulsion. Another possibility is that there may be other niche dimensions not included in my study that influence cooccurrence patterns among resprouters.

The patterns of phenotypic repulsion based on height are set against a background of environmental filtering, whereby species with similar edaphic preferences (with respect to soil nitrate concentration and $\mathrm{pH}$ ) are more likely to co-occur. This is consistent with the high degree of specialization to soil types that seems to characterize old, nutrient-impoverished landscapes in southwest Australia and elsewhere [56]. However, it is not obvious why Banksia co-occurrences should be associated with soil nitrate rather than with phosphorus, which is more likely to be 
the limiting nutrient in the highly weathered soils of southwestern Australia [56,57]. One possibility is that the measures of phosphorus concentration used here were for total phosphorus rather than plant-available forms. Unlike height, species' edaphic preferences are highly labile, so this pattern is essentially independent of relatedness, and thus leaves no signature in the phylogenetic patterns of co-occurrence. The lability of edaphic preferences is consistent with the hypothesis that local-scale specialization to soil types happens rapidly, potentially contributing to rapid speciation rates in Mediterranean-climate shrublands $[14,15,58]$.

My approach to analyzing community phylogenetic patterns based on pairwise co-occurrences is similar to that employed for schoenoid sedges in another Mediterraneanclimate shrubland, the Cape Floristic Region, which also found evidence for phylogenetic repulsion [11]. This approach differs from a recent study by Merwin et al. [12] that analyzed phylogenetic structure in Banksia communities using whole-assemblage metrics. In contrast to my finding of phylogenetic repulsion among species pairs, Merwin et al. found that many communities were phylogenetically clustered, indicating that closely-related species are more likely to co-occur. This contrast can most likely be explained as an issue of spatial scale. Their plots spanned a far greater geographical area than mine, and they interpret phylogenetic clustering as the signal of speciation and limited dispersal abilities generating many closely-related but narrowly-distributed species within a given region. In contrast, not only were my plots spread over a smaller area, but I explicitly attempted to minimize the signal of speciation history by omitting non-overlapping pairs of species from the analysis. In this way, my analyses of co-occurrence patterns were more likely to have detected local-scale ecological patterns rather than broad biogeographic effects.

Similarly, my finding of phylogenetic repulsion appears at odds with another recent study that showed that fire regeneration mode can drive phylogenetic clustering through shared adaptation of close relatives to fire-prone environments [13]. Again, the difference can be explained by a difference in scale: the study by Verdu \& Pausas [13] examined patterns across two major habitat types, one of which was fire-prone and the other not. At these scales, environmental filtering is likely to predominate over competition; furthermore, fire response in the flora they examined was phylogenetically conserved. This contrasts with Banksia in which regeneration mode is highly labile and its primary influence on community structure appears to be by mediating competitive interactions among species.

\section{Conclusions}

Phylogenies are now an integral part of community ecology, and the analysis of community structure and co- occurrence in the context of phylogenetic relatedness has contributed a great deal to our understanding of the ecological processes that govern species distributions and community assembly. However, community phylogenetic studies still commonly assume an overarching ecological structuring process for all species in a community, at a given spatial and phylogenetic scale. The different patterns for fire-killed and fire-resistant Banksia species suggest that this assumption may be simplistic, and that species with different ecological and demographic attributes, and subject to different selective pressures, can show different patterns of phylogenetic and phenotypic structure. Therefore, whole-community metrics of phylogenetic structure such as NRI and NTI may not be the most powerful way of understanding community assembly processes, and analyses of pairwise co-occurrence patterns may be more informative.

\section{Additional files}

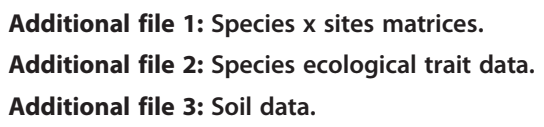

Competing interests

The author declares that he has no competing interests.

\section{Acknowledgments}

This work was funded by an Australian Research Council QEll Fellowship and Discovery Grant (DP0879971). Thanks to Geoff Kay, Lindell Bromham, Asha Cardillo and Gully Cardillo for assistance in the field, to Neil Gibson and Peter Collins for advice and logistic support, and to Lindell Bromham, Byron Lamont and Tianhua He for comments on the manuscript.

Received: 28 June 2012 Accepted: 24 September 2012

Published: 27 September 2012

\section{References}

1. Emerson BC, Gillespie RG: Phylogenetic analysis of community assembly and structure over space and time. Trends in Ecology \& Evolution 2008, 23:619-630

2. Vamosi SM, Heard SB, Vamosi JC, Webb CO: Emerging patterns in the comparative analysis of phylogenetic community structure. $\mathrm{Mol}$ Ecol 2009, 18:572-592.

3. Webb CO: Integrating phylogenies into community ecology. Ecology 2006, 87:S1-S2.

4. Webb CO, Ackerly DD, McPeek MA, Donoghue MJ: Phylogenies and community ecology. Annu Rev Ecol Syst 2002, 33:475-505.

5. Kembel SW, Hubbell SP: The phylogenetic structure of a neotropical forest tree community. Ecology 2006, 87:S86-\$99.

6. Kraft NJB, Ackerly DD: Functional trait and phylogenetic tests of community assembly across spatial scales in an Amazonian forest. Ecol Monogr 2010, 80:401-422.

7. Swenson NG, Enquist BJ, Thompson J, Zimmerman JK: The influence of spatial and size scale on phylogenetic relatedness in tropical forest communities. Ecology 2007, 88:1770-1780.

8. Helmus MR, Savage K, Diebel MW, Maxted JT, Ives AR: Separating the determinants of phylogenetic community structure. Ecol Lett 2007, 10:917-925

9. Cardillo M, Meijaard E: Phylogeny and co-occurrence of mammal species on southeast Asian islands. Glob Ecol Biogeogr 2010, 19:465-474.

10. Cavender-Bares J, Ackerly DD, Baum DA, Bazzaz FA: Phylogenetic overdispersion in Floridian oak communities. Am Nat 2004, 163:823-843. 
11. Slingsby JA, Verboom GA: Phylogenetic relatedness limits co-occurrence at fine spatial scales: Evidence from the schoenoid sedges (Cyperaceae: Schoeneae) of the Cape Floristic Region, South Africa. Am Nat 2006, 168:14-27.

12. Merwin L, He T, Lamont BB: Phylogenetic and phenotypic structure among Banksia communities in south-western Australia. J Biogeogr 2012, 39:397-407.

13. Verdu M, Pausas JG: Fire drives phylogenetic clustering in Mediterranean Basin woody plant communities. J Ecol 2007, 95:1316-1323.

14. Cowling RM, Rundel PW, Lamont BB, Arroyo MK, Arianoutsou M: Plant diversity in Mediterranean-climate regions. Trends in Ecology \& Evolution 1996, 11:362-366.

15. Cowling RM: Fire and Its Role in Coexistence and Speciation in Gondwanan Shrublands. South African Journal of Science 1987, 83:106-112.

16. Grubb PJ: The maintenance of species richness in plant communities: the importance of the regeneration niche. Biol Rev 1977, 52:107-145.

17. Lamont BB, Witkowski ETF: A test for lottery recruitment among four Banksia species based on their demography and biological attributes. Oecologia 1995, 101:299-308.

18. Miller AD, Chesson P: Coexistence in Disturbance-Prone Communities: How a Resistance-Resilience Trade-Off Generates Coexistence via the Storage Effect. Am Nat 2009, 173:E30-E43.

19. Tilman D: Resource Competition and Community Structure. Princeton: Princeton University Press; 1982.

20. Cody ML: Structural niches in plant communities. In Community Ecology. Edited by Diamond JM, Case TJ. New York: Harper \& Row; 1986:381-405.

21. Bond WJ, Midgley JJ: Ecology of sprouting in woody plants: the persistence niche. Trends in Ecology \& Evolution 2001, 16:45-51.

22. Bond WJ, Midgley JJ: The evolutionary ecology of sprouting in woody plants. Int J Plant Sci 2003, 164:S103-S114.

23. Groeneveld J, Enright NJ, Lamont BB, Wissel C: A spatial model of coexistence among three Banksia species along a topographic gradient in fire-prone shrublands. J Ecol 2002, 90:762-774.

24. Falster DS, Westoby M: Plant height and evolutionary games. Trends in ecology \& evolution (Personal edition) 2003, 18:337-343.

25. Midgley Jj: Is bigger better in plants? The hydraulic costs of increasing size in trees. Trends in Ecology \& Evolution 2003, 18:5-6.

26. Gibson N, Keighery GJ, Lyons MN, Webb A: Terrestrial flora and vegetation of the Western Australian wheatbelt. Records of the Western Australian Museum supplement 2004, 67:139-189.

27. Mast AR, Thiele K: The transfer of Dryandra R.Br. to Banksia L.f. (Proteaceae). Aust Syst Bot 2007, 20:63-71.

28. Geneious version 5.6 .3 created by Biomatters; Available from http://www. geneious.com/.

29. Drummond AJ, Rambaut A: BEAST: Bayesian evolutionary analysis by sampling trees. BMC Evol Biol 2007, 7:214

30. Crisp M, Cook L, Steane D: Radiation of the Australian flora: what can comparisons of molecular phylogenies across multiple taxa tell us about the evolution of diversity in present-day communities? Phil Trans $R$ SoC Lond B 2004, 359:1551-1571.

31. Sauquet $H$, Weston $P H$, Anderson CL, Barker NP, Cantrill DJ, Mast AR, Savolainen $\mathrm{V}$ : Contrasted patterns of hyperdiversification in Mediterranean hotspots. Proc Natl Acad Sci U S A 2009, 106:221-225.

32. Drummond AJ, Ho SYW, Phillips MJ, Rambaut A: Relaxed Phylogenetics and Dating with Confidence. PLOS Biol 2006, 4:e88.

33. Schliep KP: phangorn: phylogenetic analysis in R. Bioinformatics 2011, 27:592-593.

34. George AS: Banksia. Flora of Australia 1999, 17B:175-250.

35. George AS: Dryandra. Flora of Australia 1999, 17B:251-363.

36. Cavanagh T, Pieroni M: The Dryandras. Hawthorn, Victoria: Australian Plants Society Inc;; 2006.

37. Moles AT, Ackerly DD, Webb CO, Tweddle JC, Dickie JB, Pitman AJ, Westoby M: Factors that shape seed mass evolution. Proc Natl Acad Sci U S A 2005, 102:10540-10544.

38. Westoby M, Falster DS, Moles AT, Vesk PA, Wright IJ: Plant ecological strategies: some leading dimensions of variation between species. Annu Rev Ecol Syst 2002, 33:125-159.

39. Westoby M, Leishman M, Lord J: Comparative ecology of seed size and dispersal. Philosophical Transactions of the Royal Society of London Series B-Biological Sciences 1996, 351:1309-1317.
40. Craine JM, Wolkovich EM, Towne EG, Kembel SW: Flowering phenology as a functional trait in a tallgrass prairie. New Phytol 2011, 193:673-682.

41. Rathcke B, Lacey EP: Phenological patterns of terrestrial plants. Annu Rev Ecol Syst 1985, 16:179-214.

42. Hopper SD, Gioia P: The Southwest Australian Floristic Region: Evolution and conservation of a global hot spot of biodiversity. Annu Rev Ecol Syst 2004, 35:623-650.

43. Cowling RM, Witkowski ETF, Milewski AV, Newbey KR: Taxonomic, Edaphic and Biological Aspects of Narrow Plant Endemism on Matched Sites in Mediterranean South-Africa and Australia. J Biogeogr 1994, 21:651-664.

44. FloraBase: http://florabase.calm.wa.gov.au/.

45. ESRI: ArcGIS Version 9. In Book ArcGIS Version 9. City: Environmental Systems Research Institute, Inc; 2009.

46. Schoener TW: Nonsynchronous spatial overlap of lizards in patchy habitats. Ecology 1970, 51:408.

47. Kembel SW, Cowan PD, Helmus MR, Cornwell WK, Morlon H, Ackerly DD, Blomberg SP, Webb CO: Picante: R tools for integrating phylogenies and ecology. Bioinformatics 2010, 26:1463-1464.

48. Pagel MD: Inferring the historical patterns of biological evolution. Nature 1999, 401:877-884.

49. Fritz SA, Purvis A: Selectivity in Mammalian Extinction Risk and Threat Types: a New Measure of Phylogenetic Signal Strength in Binary Traits. Conserv Biol 2010, 24:1042-1051.

50. Felsenstein J: Phylogenies and the comparative method. Am Nat 1985, 125:1-15.

51. Garland T, Harvey PH, Ives AR: Procedures for the analysis of comparative data using phylogenetically independent contrasts. Syst Biol 1992 41:18-32.

52. Bond WJ, Cowling RM, Richards MB: Competition and coexistence. In The Ecology of Fynbos: Nutrients, Fire and Diversity. Edited by Cowling RM. Oxford: Oxford University press; 1992:206-225.

53. Midgley JJ: Why the world's vegetation is not totally dominated by resprouting plants; Because resprouters are shorter than reseeders. Ecography 1996, 19:92-95.

54. Pronk TE, Schieving F, Anten NPR, Werger MJA: Plants that differ in height investment can coexist if they are distributing non-uniformly within an area. Ecol Complex 2007, 4:182-191.

55. Lamont BB, Markey A: Biogeography of fire-killed and resprouting Banksia species in south-western Australia. Aust J Bot 1995, 43:283-303.

56. Lambers H, Brundrett MC, Raven JA, Hopper SD: Plant mineral nutrition in ancient landscapes: high plant species diversity on infertile soils is linked to functional diversity for nutritional strategies. Plant Soil 2010, 348:7-27.

57. Lambers H, Raven JA, Shaver GR, Smith SE: Plant nutrient-acquisition strategies change with soil age. Trends in Ecology \& Evolution 2008, 23:95-103.

58. Rajakaruna N: The edaphic factor in the origin of plant species. Int Geol Rev 2004, 46:471-478.

doi:10.1186/1472-6785-12-21

Cite this article as: Cardillo: The phylogenetic signal of species cooccurrence in high-diversity shrublands: different patterns for fire-killed and fire-resistant species. BMC Ecology 2012 12:21.

\section{Submit your next manuscript to BioMed Central and take full advantage of:}

- Convenient online submission

- Thorough peer review

- No space constraints or color figure charges

- Immediate publication on acceptance

- Inclusion in PubMed, CAS, Scopus and Google Scholar

- Research which is freely available for redistribution 